\title{
Diagnóstico da cavidade óssea idiopática: revisão sistematizada da literatura
} Diagnosis of the idiopathic bone cavity: a systematic literature review

Diagnóstico de la cavidad ósea idiopática: revisión sistemática de la literatura

Recebido: 16/08/2020 | Revisado: 27/08/2020 | Aceito: 15/09/2020 | Publicado: 16/09/2020

\section{Vinícius Lima de Almeida}

ORCID: https://orcid.org/0000-0002-2135-1040

Hospital de Clínicas da Universidade Federal de Uberlândia, Brasil

E-mail: viniciusl.dealmeida91@gmail.com

Cristóvão Marcondes de Castro Rodrigues

ORCID: https://orcid.org/0000-0002-0590-9075

Hospital de Clínicas da Universidade Federal de Uberlândia, Brasil

E-mail: cristovao-marcondes@hotmail.com

Marco Túllio Brazão-Silva

ORCID: https://orcid.org/0000-0003-1957-949X

Universidade Estadual de Montes Claros, Brasil

E-mail: marcotullio@gmail.com

Flávio de Freitas Bueno

ORCID: https://orcid.org/0000-0003-0699-1127

Instituto de Educação Imed-Montes Claros, Brasil

E-mail: diretoriaflavioimed@gmail.com

Maiolino Thomaz Fonseca Oliveira

ORCID: https://orcid.org/0000-0002-7800-6571

Hospital de Urgências de Goiânia, Brasil

E-mail: maiolinothomaz@ hotmail.com

\section{Resumo}

Introdução: A cavidade óssea idiopática é uma lesão de aparência radiográfica cística que pode acometer qualquer osso. Especialmente em ossos maxilares representa cerca de $1 \%$ das lesões císticas, um dado que pode estar subestimado visto que a biópsia e o diagnóstico clínico podem ser dispensados dados o curso e características da lesão. Metdologia: A 
presente revisão sistematizada da literatura foi realizada por meio de busca nas bases de dados PubMed, SciELO, LILACS e OpenGrey. Resultados: Dentre os 21 estudos inclusos, a maioria foi de coorte retrospectivo, com amostra total de 401 pacientes, idade mínima de 7 anos e máxima de 75 anos. A localização das lesões ocorreu de acordo com o seguinte quantitativo de pacientes: região posterior de mandíbula (268), região anterior de mandíbula (104) e maxila (30). Em 40 pacientes houve expansão óssea. Com relação às características radiográficas, 369 pacientes apresentaram lesões com distribuição unilateral e 29 com localização bilateral, enquanto o padrão unilocular esteve presente em 97 e o padrão multilocular em 23 pacientes. Conclusão: a cavidade óssea idiopática encontra-se de forma predominante durante as primeiras duas décadas de vida em pacientes de ambos os sexos, tem maior incidência em região posterior de mandíbula, é geralmente assintomática, radiograficamente apresentando-se de forma incisiva como uma lesão radiolúcida unilocular com ar, sangue ou líquido seroso no seu interior, tendo a exploração cirúrgica e curetagem como tratamento de escolha e baixo índice de recidiva.

Palavras-chave: Cavidade óssea idiopática; Cisto ósseo simples; Diagnóstico; Odontologia geral.

\section{Abstract}

Introduction: The idiopathic bone cavity is a lesion with a cystic radiographic appearance that can affect any bone. Especially in maxillary bones it represents about $1 \%$ of cystic lesions, a fact that can be underestimated since biopsy and clinical diagnosis can be dispensed with given the course and characteristics of the lesion. Methodology: This systematic review of the literature was carried out by searching the PubMed, SciELO, LILACS and OpenGrey databases. Results: Among the 21 studies included, most were from a retrospective cohort, with a total sample of 401 patients, with a minimum age of 7 years and a maximum age of 75 years. The lesions were located according to the following number of patients: posterior mandible region (268), anterior mandible region (104) and maxilla (30). In 40 patients there was bone expansion. Regarding radiographic characteristics, 369 patients presented lesions with unilateral distribution and 29 with bilateral location, while the unilocular pattern was present in 97 and the multilocular pattern in 23 patients. Conclusion: the idiopathic bone cavity is predominantly found during the first two decades of life in patients of both sexes, has a greater incidence in the posterior region of the mandible, is generally asymptomatic, radiographically presenting itself incisively as a radiolucent lesion. unilocular with air, blood 
or serous liquid inside, with surgical exploration and curettage as the treatment of choice and a low recurrence rate.

Keywords: Idiopathic bone cavity; Simple bone cyst; Diagnosis; General dentistry.

\section{Resumen}

Introducción: La cavidad ósea idiopática es una lesión de aspecto quístico radiográfico que puede afectar a cualquier hueso. Especialmente en los maxilares representa alrededor del 1\% de las lesiones quísticas, hecho que puede subestimarse ya que se puede prescindir de la biopsia y el diagnóstico clínico dado el curso y las características de la lesión. Metodología: Esta revisión sistemática de la literatura se realizó mediante búsquedas en las bases de datos PubMed, SciELO, LILACS y OpenGrey. Resultados: De los 21 estudios incluidos, la mayoría fueron de una cohorte retrospectiva, con una muestra total de 401 pacientes, con una edad mínima de 7 años y una edad máxima de 75 años. Las lesiones se localizaron según el siguiente número de pacientes: región posterior de la mandíbula (268), región anterior de la mandíbula (104) y maxilar (30). En 40 pacientes hubo expansión ósea. En cuanto a las características radiográficas, 369 pacientes presentaron lesiones de distribución unilateral y 29 de localización bilateral, mientras que el patrón unilocular estuvo presente en 97 y el patrón multilocular en 23 pacientes. Conclusión: la cavidad ósea idiopática se encuentra predominantemente durante las dos primeras décadas de la vida en pacientes de ambos sexos, tiene mayor incidencia en la región posterior de la mandíbula, es generalmente asintomática, presentándose radiográficamente de forma incisiva como una lesión radiolúcida. unilocular con aire, sangre o líquido seroso en su interior, con exploración quirúrgica y legrado como tratamiento de elección y baja tasa de recurrencia.

Palabras clave: Cavidad ósea idiopática; Quiste óseo simple; Diagnóstico; Odontología general.

\section{Introdução}

Cavidade óssea idiopática (COI) é uma lesão intraóssea comum na metáfise de ossos longos, relativamente raro nos ossos maxilares, representando aproximadamente $1 \%$ de todos os cistos mandibulares (Harnet et al., 2008). Inicialmente descrita como cisto ósseo traumático, apresenta-se como uma cavidade óssea não recoberta por epitélio, vazia ou parcialmente preenchida com líquido seroso ou sanguinolento (Lucas, 1929; Rushton, 1946). Indivíduos com faixa etária entre a primeira e segunda década apresentam maior 
predisposição para o desenvolvimento desse tipo de lesão, não havendo predileção sexual (Tong, Ng \& Yan, 2003). Embora, os resultados do estudo de An et al., (2014), mostrem maior incidência em pacientes do sexo feminino.

Várias teorias foram sugeridas para sua etiopatogenia, tais como: anormalidades vasculares intra-ósseas, degeneração de tumores ósseos, trauma e alteração do metabolismo ósseo (Blum,1955; Whinery, 1955). Dessa forma, recebe denominações como cisto ósseo simples, cisto ósseo hemorrágico, cisto ósseo solitário ou cisto ósseo de câmara única (Lucas, 1929).

Raramente as lesões encontram-se na maxila, localizando-se ocasionalmente na sínfise mandibular e principalmente na região de corpo e ramo mandibular (Forssell, Forssell, Happonen, \& Neva, 1988). Não há acometimento da vitalidade, reabsorção radicular, estímulo à mobilidade ou deslocamento das unidades dentais envolvidas na lesão (MartinsFilho et al., 2012). Apesar de geralmente serem assintomáticas, pode ocorrer dor em 10\% a $30 \%$ dos pacientes, além da possibilidade de haver parestesia, fístulas, retardo na erupção dental, edema intra-oral e extra-oral e fratura patológica da mandíbula (Shimoyama et al., 1999).

Ao exame de imagem, a COI apresenta-se como uma cavidade radiolúcida bem circunscrita, geralmente unilocular e com margens escleróticas. Pode estender-se para os septos interdentais e, em alguns casos, ser multilocular e assemelhar-se a outras entidades patológicas (Cowan, 1980). O diagnóstico diferencial pode incluir lesões como cisto dentígero, tumor odontogênico queratocístico, mixoma odontogênico, ameloblastoma, cisto radicular e lesão central de células gigantes, cisto ósseo aneurismático, defeito focal da medula óssea e malformação vascular (Cowan, 1980; Shigematsu, Fujita, \& Watanabe, 1994).

Devido a escassez de material intra-lesional para biópsia, o diagnóstico histopatológico pode ser dificultado. Portanto, o diagnóstico definitivo pode basear-se na experiência clínica e guiar a escolha das opções de tratamento (Lindsay, Martin, \& Green, 1966). Dentre as terapêuticas cirúrgicas disponíveis, a curetagem das paredes ósseas é o método de escolha (Suei, Taguchi, \& Tanimoto, 2007). Outras modalidades de tratamento incluem inserção de enxerto ósseo ou hidroxiapatita ou mesmo injeção de sangue autólogo na cavidade óssea, embora a auto-resolução possa ocorrer (Matsumara et al., 1998; Wakasa, Kawai, Aiga, \& Kishi, 2002). Instituído o tratamento, o acompanhamento clínico e radiográfico deve prosseguir por pelo menos 6 a 12 meses, apesar de casos de recidiva terem ocorrido em 3 anos após a cirurgia. (Tsukamoto, Sasaki, Mese, \& Matsumara, 2003). 
Research, Society and Development, v. 9, n. 10, e109107656, 2020

(CC BY 4.0) | ISSN 2525-3409 | DOI: http://dx.doi.org/10.33448/rsd-v9i10.7656

Em virtude da importância de basear-se em evidências científicas para a adequada condução clínica, o presente estudo objetiva realizar uma revisão sistematizada da literatura abordando características clínico-patológicas e o manejo da cavidade óssea idiopática em ossos gnáticos.

\section{Metodologia}

A presente revisão sistematizada da literatura trata-se de um estudo quantitativo realizado por meio da busca nas seguintes bases de dados eletrônicas: PubMed, SciELO, Latin American and Caribbean Health Sciences (LILACS) e OpenGrey. Especificamente, o banco de dados OpenGrey foi usado para pesquisar a literatura cinza e evitar possíveis vieses de seleção. O Medical Subject Headings (MeSH) foi utilizado para selecionar os descritores controlados. Operadores booleanos (OR e AND) foram utilizados para combinar os descritores. A Tabela 1 mostra a estratégia de busca selecionada.

Tabela 1- Estratégia de busca.

\begin{tabular}{|c|c|c|}
\hline Database & Search Strategy (Fevereiro, 2019) & Results \\
\hline \multirow[t]{5}{*}{$\begin{array}{l}\text { PubMed } \\
\text { http://www.ncbi.nlm.nih.gov/pubmed }\end{array}$} & $\begin{array}{l}\text { "Traumatic"[All Fields] AND "bone cyst"[All } \\
\text { Fields] AND "Maxilla"[All Fields] }\end{array}$ & 178 \\
\hline & $\begin{array}{l}\text { "Idiopathic"[All Fields] AND "bone cyst"[All } \\
\text { Fields] AND "Maxilla"[All Fields] }\end{array}$ & 59 \\
\hline & $\begin{array}{l}\text { "Hemorrhagic"[All Fields] AND "bone } \\
\text { cyst"[All Fields] AND "Maxilla"[All Fields] }\end{array}$ & 93 \\
\hline & $\begin{array}{l}\text { "Solitary"[All Fields] AND "bone cyst"[All } \\
\text { Fields] AND "Maxilla"[All Fields] }\end{array}$ & 174 \\
\hline & $\begin{array}{l}\text { "Simple"[All Fields] AND "bone cyst"[All } \\
\text { Fields] AND "Maxilla"[All Fields] }\end{array}$ & 194 \\
\hline \multirow{5}{*}{$\begin{array}{l}\text { LILACS } \\
\text { http://lilacs.bvsalud.org/ }\end{array}$} & $\begin{array}{l}\text { simple AND bone AND cyst AND ( } \\
\mathrm{db}:(" L I L A C S "))\end{array}$ & 53 \\
\hline & $\begin{array}{l}\text { traumatic AND bone AND cyst AND ( } \\
\text { db:("LILACS")) }\end{array}$ & 43 \\
\hline & $\begin{array}{l}\text { solitary AND bone AND cyst AND ( } \\
\text { db:("LILACS")) }\end{array}$ & 158 \\
\hline & $\begin{array}{l}\text { idiopathic AND bone AND cyst AND ( } \\
\text { db:("LILACS")) }\end{array}$ & 12 \\
\hline & $\begin{array}{l}\text { hemorrhagic AND bone AND cyst AND ( } \\
\text { db:("LILACS")) }\end{array}$ & 6 \\
\hline \multirow{5}{*}{$\begin{array}{l}\text { SciELO } \\
\text { http://www.scielo.org/ }\end{array}$} & Simple AND Bone AND Cyst & 17 \\
\hline & Traumatic AND Bone AND Cyst & 12 \\
\hline & Solitary AND Bone AND Cyst & 15 \\
\hline & Idiopathic AND Bone AND Cyst & 7 \\
\hline & Hemorrhagic AND Bone AND Cyst & 6 \\
\hline \multirow{5}{*}{$\begin{array}{l}\text { OpenGrey } \\
\text { http://www.opengrey.eu/ }\end{array}$} & Simple AND Bone AND Cyst & 0 \\
\hline & Traumatic AND Bone AND Cyst & 0 \\
\hline & Solitary AND Bone AND Cyst & 0 \\
\hline & Idiopathic AND Bone AND Cyst & 0 \\
\hline & Hemorrhagic AND Bone AND Cyst & 0 \\
\hline TOTAL & & 1027 \\
\hline
\end{tabular}

Fonte: Tabela confeccionada pelos autores. 
Research, Society and Development, v. 9, n. 10, e109107656, 2020

(CC BY 4.0) | ISSN 2525-3409 | DOI: http://dx.doi.org/10.33448/rsd-v9i10.7656

Na Tabela 1 é possível observar a estratégia de busca lançada em cada base de dados, constituindo-se de descritores selecionados por meio do MeSH e combinados por meio de operadores boleanos.

A coleta de dados foi realizada em diferentes momentos. Primeiro todas as referências foram exportadas para o software Mendeley Deskyop 1.13.3 (Mendeley Ltd., Londres, Inglaterra), a fim de rastrear possíveis registros duplicados. Posteriormente, os títulos e resumos foram lidos detalhadamente para excluir artigos fora do escopo da pesquisa. Nesta fase, foram excluídos artigos de revisão da literatura e relatos isolados de casos. Foram inclusos estudos classificados como série de casos e coorte retrospectivo que abordam características clínico-patológicas e o manejo da cavidade óssea idiopática em ossos gnáticos. Não houve restrição de idioma e nem quanto ao período de publicação. Os artigos em que o título e o resumo não apresentavam informações suficientes tiveram o texto completo analisado, a fim de decidir sobre sua elegibilidade. Aqueles que apresentaram um título dentro do tema, mas os resumos não estavam disponíveis, também foram obtidos e analisados na íntegra. Artigos de interesse, presentes nas referências de estudos inclusos, e que contemplaram os critérios de elegibilidade, também foram inclusos no presente estudo. Essas avaliações foram realizadas independentemente por dois revisores dos critérios de elegibilidade (V.L.A. e C.M.C.R.).

Com relação aos artigos inclusos, foram extraídos os seguintes dados: autor, País de realização do estudo e data de publicação, tipo de estudo, amostra, idade, tipo de lesão, histórico de trauma orofacial, sintomatologia, localização das lesões, presença de aumento volumétrico em ossos faciais acometidos, características radiográficas (unilateral, bilateral, unilocular, multilocular), achados intra-operatórios, tratamento e recidiva.

\section{Resultados}

O fluxograma apresentado na Figura 1 demonstra nosso esquema de seleção, enquanto a Tabela 2 apresenta o resumo das características dos estudos inclusos na presente revisão. 
(CC BY 4.0) | ISSN 2525-3409 | DOI: http://dx.doi.org/10.33448/rsd-v9i10.7656

Figura 1- Fluxograma demonstrando o esquema de seleção dos estudos.

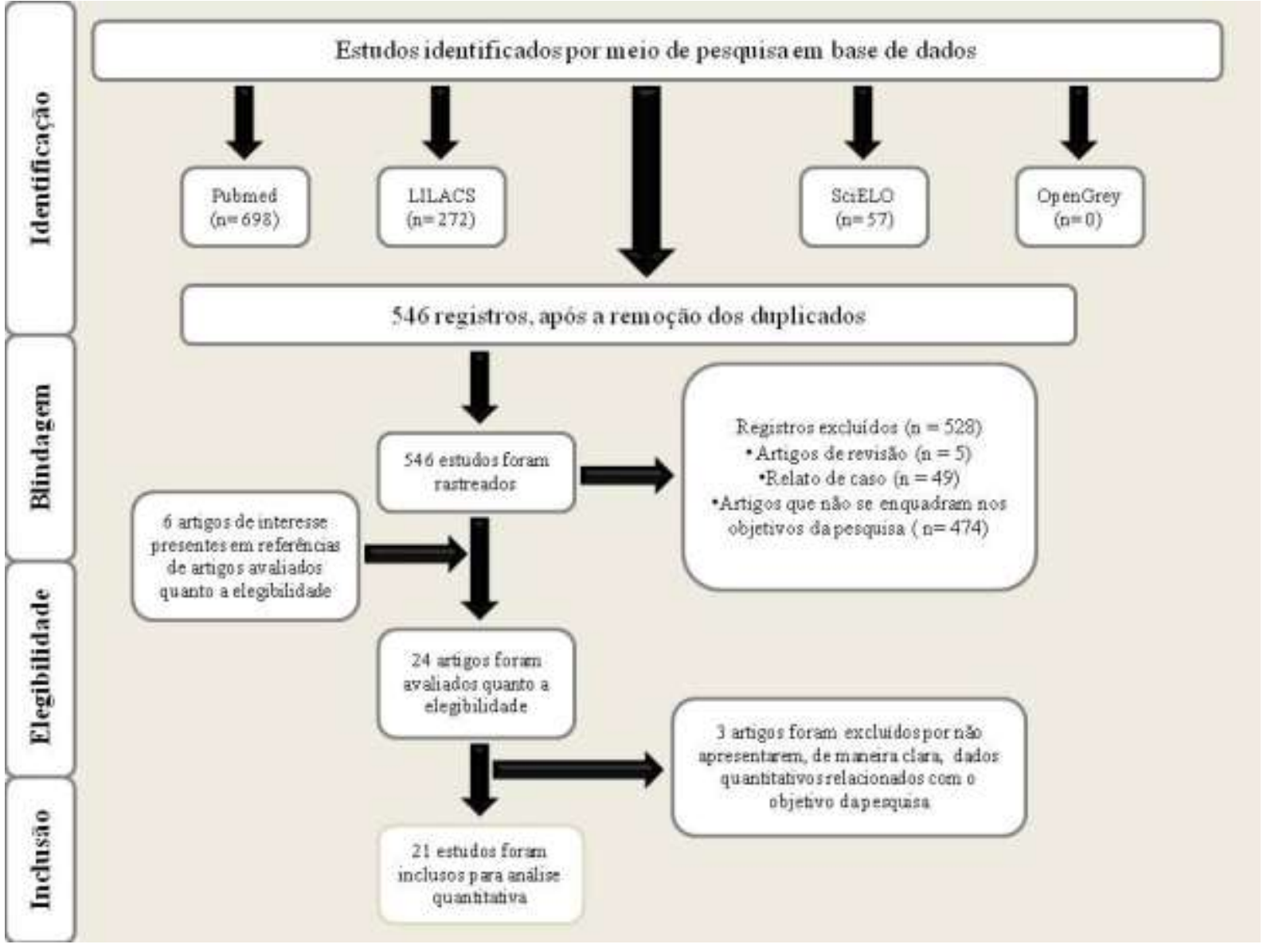

Fonte: Imagem confeccionada pelos autores.

De acordo com a Figura 1 é possível observar o esquema de seleção, desde a obtenção inicial do quantitativo de artigos dentre os quais foram removidos os duplicados, excluídos os estudos que não contemplaram o escopo da presente revisão e selecionados os artigos que cumpriram os critérios de inclusão. 
Research, Society and Development, v. 9, n. 10, e109107656, 2020

(CC BY 4.0) | ISSN 2525-3409 | DOI: http://dx.doi.org/10.33448/rsd-v9i10.7656

Tabela 2- Características dos estudos inclusos.

\begin{tabular}{|c|c|c|c|c|c|c|c|c|c|c|c|c|c|c|}
\hline \multirow{2}{*}{$\begin{array}{c}\text { Autor, ano e } \\
\text { País de } \\
\text { publicação }\end{array}$} & \multirow{2}{*}{$\begin{array}{l}\text { Tipo de } \\
\text { estudo }\end{array}$} & \multirow[t]{2}{*}{ Amostra } & \multirow{2}{*}{$\begin{array}{l}\text { Idade } \\
\text { (Anos) }\end{array}$} & \multirow[t]{2}{*}{ Tipo de lesão } & \multirow{2}{*}{$\begin{array}{l}\text { Histórico de } \\
\text { trauma }\end{array}$} & \multirow[t]{2}{*}{ Sintomatologia } & \multirow[t]{2}{*}{ Localização } & \multirow{2}{*}{$\begin{array}{l}\text { Expansão de } \\
\text { cortical óssea }\end{array}$} & \multicolumn{4}{|c|}{ Características Radiográficas } & \multirow[t]{2}{*}{ Tratamento } & \multirow{2}{*}{$\begin{array}{l}\text { Conteúdo } \\
\text { intra-lesional }\end{array}$} \\
\hline & & & & & & & & & Unilateral & Bilateral & Unilocular & Multilocular & & \\
\hline $\begin{array}{c}\text { Hansen, } \\
\text { Sapone, \& } \\
\text { Sproat (1974) } \\
\text { Estados } \\
\text { Unidos }\end{array}$ & $\begin{array}{c}\text { Coorte } \\
\text { retrospectivo }\end{array}$ & $\begin{array}{c}65 \\
\left(32 \mathrm{o}^{1} \mathrm{e} 33 \text { 우 }\right.\end{array}$ & 7-75 & $\begin{array}{l}\text { Cisto ósseo } \\
\text { traumático }\end{array}$ & 38 & 6 & $\begin{array}{c}\text { Mandíbula } \\
\text { (RP): } 26 \\
\text { (RA): } 18 \\
\text { Maxila: } 21\end{array}$ & 14 & 65 & 0 & * & * & $\begin{array}{l}\text { Exploração } \\
\text { cirúrgica }\end{array}$ & $\begin{array}{c}\text { Cavidade } \\
\text { vazia, líquido } \\
\text { seroso e sangue }\end{array}$ \\
\hline $\begin{array}{c}\text { Beasley } \\
(1979) \\
\text { Estados } \\
\text { Unidos } \\
\end{array}$ & $\begin{array}{c}\text { Coorte } \\
\text { retrospectivo }\end{array}$ & $\begin{array}{c}26 \\
\left(16 \mathrm{O}^{7} \mathrm{e} 10 \text { q }\right.\end{array}$ & $12-57$ & $\begin{array}{l}\text { Cisto ósseo } \\
\text { traumático }\end{array}$ & 7 & 6 & $\begin{array}{c}\text { Mandíbula } \\
\text { (RP): } 20 \\
\text { (RA): } 3 \\
\text { Maxila: } 4\end{array}$ & * & 25 & 1 & * & * & $\begin{array}{l}\text { Exploração } \\
\text { cirúrgica e } \\
\text { curetagem }\end{array}$ & $\begin{array}{l}\text { Cavidade vazia } \\
\text { e sangue }\end{array}$ \\
\hline $\begin{array}{c}\text { Davis Junior, } \\
\text { Buchs, \& } \\
\text { Davis (1979) } \\
\text { Estados } \\
\text { Unidos }\end{array}$ & $\begin{array}{c}\text { Coorte } \\
\text { retrospectivo }\end{array}$ & $\begin{array}{c}15 \\
\left(7 \mathrm{o}^{7} \mathrm{e} 8 \text { P }\right)\end{array}$ & $11-58$ & $\begin{array}{c}\text { Cisto de } \\
\text { extravasamento }\end{array}$ & * & * & $\begin{array}{c}\text { Mandíbula } \\
\text { (RP): } 12 \\
\text { (RA):3 }\end{array}$ & * & 14 & 1 & * & * & $\begin{array}{l}\text { Exploração } \\
\text { cirúrgica e } \\
\text { curetagem }\end{array}$ & Sangue \\
\hline $\begin{array}{c}\text { Kaugars \& } \\
\text { Cale (1987) } \\
\text { Estados } \\
\text { Unidos }\end{array}$ & $\begin{array}{c}\text { Coorte } \\
\text { retrospectivo }\end{array}$ & $\begin{array}{c}67 \\
(33 \text { o e } 34 \text { ㅇ })\end{array}$ & 9-68 & $\begin{array}{l}\text { Cisto ósseo } \\
\text { traumático }\end{array}$ & 6 & 10 & $\begin{array}{c}\text { Mandíbula } \\
\text { (RP): } 48 \\
\text { (RA): } 18 \\
\text { Maxila: } 1\end{array}$ & * & 51 & 13 & * & * & * & * \\
\hline $\begin{array}{c}\text { Forssell et al. } \\
\text { (1988) } \\
\text { Finlândia } \\
\end{array}$ & $\begin{array}{c}\text { Coorte } \\
\text { retrospectivo }\end{array}$ & $\begin{array}{c}22 \\
\left(13{ }^{A} \mathrm{e} 9 \rho\right)\end{array}$ & $8-59$ & $\begin{array}{l}\text { Cisto ósseo } \\
\text { simples }\end{array}$ & 4 & 5 & $\begin{array}{c}\text { Mandíbula } \\
\text { (RP): } 16 \\
\text { (RA):6 }\end{array}$ & 1 & 21 & 1 & 20 & 2 & $\begin{array}{l}\text { Exploração } \\
\text { cirúrgica e } \\
\text { curetagem }\end{array}$ & $\begin{array}{c}\text { Cavidade } \\
\text { vazia, líquido } \\
\text { seroso e sangue }\end{array}$ \\
\hline $\begin{array}{l}\text { Matsumura et } \\
\text { al. (1998) } \\
\text { Japão }\end{array}$ & $\begin{array}{c}\text { Coorte } \\
\text { retrospectivo }\end{array}$ & $\begin{array}{c}51 \\
\left(23{ }^{\lambda} \mathrm{e} 28 \text { 우 }\right.\end{array}$ & $9-53$ & $\begin{array}{l}\text { Cisto ósseo } \\
\text { simples }\end{array}$ & 6 & 7 & $\begin{array}{c}\text { Mandíbula } \\
\text { (RP): } 46 \\
\text { (RA):4 } \\
\text { Maxila: } 3\end{array}$ & 12 & 49 & 2 & * & * & * & * \\
\hline $\begin{array}{c}\text { Peñarrocha- } \\
\text { Diago, } \\
\text { Sanchis- } \\
\text { Bielsa, Bonet- } \\
\text { Marco, \& } \\
\text { Minguez- } \\
\text { Sanz, (2001) } \\
\text { Espanha }\end{array}$ & Série de casos & $\left(2 \delta^{7} \mathrm{e} 5\right.$ 운 & 9-29 & $\begin{array}{l}\text { Cisto ósseo } \\
\text { simples }\end{array}$ & 1 & 0 & $\begin{array}{l}\text { Mandíbula } \\
\text { (RP): } 2 \\
\text { (RA):5 }\end{array}$ & * & 7 & 0 & 7 & 0 & $\begin{array}{l}\text { Exploração } \\
\text { cirúrgica, } \\
\text { curetagem e } \\
\text { enxertia }\end{array}$ & * \\
\hline $\begin{array}{c}\text { Cortell- } \\
\text { Ballester, } \\
\text { Figueiredo, } \\
\text { Beirini-Aytés, } \\
\& \text { Gay-Escoda } \\
(2009) \\
\text { Espanha } \\
\end{array}$ & $\begin{array}{c}\text { Coorte } \\
\text { retrospectivo }\end{array}$ & $\left(7 \begin{array}{c}21 \\
\mathrm{e} 14\end{array}\right.$ & $8-45$ & $\begin{array}{l}\text { Cisto ósseo } \\
\text { traumático }\end{array}$ & 5 & 0 & $\begin{array}{c}\text { Mandíbula } \\
\text { (RP): } 18 \\
\text { (RA):3 }\end{array}$ & * & 21 & 0 & 21 & 0 & $\begin{array}{l}\text { Exploração } \\
\text { cirúrgica e } \\
\text { curetagem }\end{array}$ & $\begin{array}{c}\text { Cavidade vazia } \\
\text { e tecido } \\
\text { conjuntivo }\end{array}$ \\
\hline $\begin{array}{l}\text { Paes, Santos, } \\
\text { Marucci, } \\
\text { Costa, \& }\end{array}$ & Série de casos & $\begin{array}{c}6 \\
\left(50^{-1} \mathrm{e} 1 \text { 19) }\right.\end{array}$ & $12-24$ & $\begin{array}{l}\text { Cisto ósseo } \\
\text { simples }\end{array}$ & 2 & 0 & $\begin{array}{l}\text { Mandíbula } \\
\text { (RP): } 5 \\
\text { (RA): } 1\end{array}$ & * & 6 & 0 & 6 & 0 & * & Cavidade vazia \\
\hline
\end{tabular}


Research, Society and Development, v. 9, n. 10, e109107656, 2020

(CC BY 4.0) | ISSN 2525-3409 | DOI: http://dx.doi.org/10.33448/rsd-v9i10.7656

\begin{tabular}{|c|c|c|c|c|c|c|c|c|c|c|c|c|c|c|}
\hline $\begin{array}{c}\text { Oliveira } \\
(2010) \\
\text { Brasil }\end{array}$ & & & & & & & & & & & & & & \\
\hline $\begin{array}{c}\text { Discacciati et } \\
\text { al. (2012) } \\
\text { Brasil }\end{array}$ & $\begin{array}{c}\text { Coorte } \\
\text { retrospectivo }\end{array}$ & 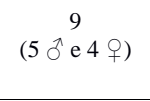 & $10-17$ & $\begin{array}{c}\text { Cavidade óssea } \\
\text { idiopática }\end{array}$ & 6 & 0 & $\begin{array}{l}\text { Mandíbula } \\
\text { (RP): } 3 \\
\text { (RA):6 }\end{array}$ & 0 & 8 & 1 & $*$ & $*$ & $\begin{array}{l}\text { Exploração } \\
\text { cirúrgica e } \\
\text { curetagem }\end{array}$ & $\begin{array}{c}\text { Cavidade } \\
\text { vazia, líquido } \\
\text { seroso e sangue }\end{array}$ \\
\hline $\begin{array}{l}\text { Martins-Filho } \\
\text { et al. (2012) } \\
\text { Brasil }\end{array}$ & $\begin{array}{c}\text { Coorte } \\
\text { retrospectivo }\end{array}$ & $\begin{array}{c}26 \\
(17 \overbrace{}^{\lambda} \text { e } 9 \text { 우 })\end{array}$ & $10-70$ & $\begin{array}{l}\text { Cisto ósseo } \\
\text { traumátio }\end{array}$ & 4 & 3 & $\begin{array}{l}\text { Mandíbula } \\
\text { (RP): } 18 \\
\text { Mandíbula } \\
\text { (RA): } 8\end{array}$ & 3 & 25 & 1 & 19 & 7 & $\begin{array}{l}\text { Exploração } \\
\text { cirúrgica e } \\
\text { curetagem }\end{array}$ & $\begin{array}{c}\text { Ar, líquido } \\
\text { seroso e } \\
\text { serossanguíneo }\end{array}$ \\
\hline $\begin{array}{c}\text { Sabino- } \\
\text { Bezerra, } \\
\text { Santos-Silva, } \\
\text { Jorge, Gouvêa, } \\
\& \text { Lopes } \\
(2013) \\
\text { Brasil } \\
\end{array}$ & Série de casos & 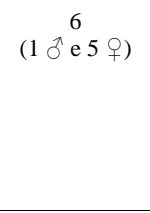 & 13- 45 & $\begin{array}{l}\text { Cisto ósseo } \\
\text { simples }\end{array}$ & 0 & 1 & $\begin{array}{l}\text { Mandíbula } \\
\text { (RP): } 4 \\
\text { (RA): } 2\end{array}$ & 3 & 6 & 0 & 2 & 4 & $\begin{array}{l}\text { Exploração } \\
\text { cirúrgica e } \\
\text { curetagem }\end{array}$ & Cavidade vazia \\
\hline $\begin{array}{c}\text { Urs, } \\
\text { Augustine, \& } \\
\text { Chawla (2014) } \\
\text { India }\end{array}$ & $\begin{array}{c}\text { Coorte } \\
\text { retrospectivo }\end{array}$ & $\left(3 \delta^{7} \mathrm{e}^{7}\right.$ ㅇ $)$ & $10-50$ & $\begin{array}{l}\text { Cisto ósseo } \\
\text { traumático }\end{array}$ & $*$ & 2 & $\begin{array}{l}\text { Mandíbula } \\
\text { (RP): } 5 \\
\text { (RA): } 2\end{array}$ & 2 & 7 & 0 & 2 & 5 & $\begin{array}{l}\text { Exploração } \\
\text { cirúrgica }\end{array}$ & * \\
\hline $\begin{array}{c}\text { An et al. } \\
(2014) \\
\text { República da } \\
\text { Coréia }\end{array}$ & Série de casos & 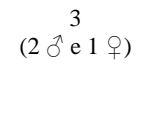 & $11-18$ & $\begin{array}{l}\text { Cisto ósseo } \\
\text { simples }\end{array}$ & $*$ & 0 & $\begin{array}{l}\text { Mandíbula } \\
\text { (RP): } 1 \\
\text { (RA):3 }\end{array}$ & 1 & 0 & 3 & 3 & 0 & $\begin{array}{l}\text { Exploração } \\
\text { cirúrgica e } \\
\text { curetagem }\end{array}$ & Cavidade vazia \\
\hline $\begin{array}{c}\text { Kumar, } \\
\text { Sherubin, } \\
\text { Raman, \& } \\
\text { Shettar (2014) } \\
\text { India }\end{array}$ & Série de casos & $\left(2 \mathrm{~s}^{2} \mathrm{e} 0\right.$ o p $)$ & $15-21$ & $\begin{array}{l}\text { Cisto ósseo } \\
\text { traumático }\end{array}$ & $*$ & 2 & $\begin{array}{l}\text { Mandíbula } \\
\text { (RP): } 2 \\
\text { (RA): } 0\end{array}$ & 2 & 0 & 2 & 1 & 1 & \begin{tabular}{|c|} 
Exploração \\
cirúrgica, \\
ressecção \\
marginal de \\
mandibula e \\
reconstrução \\
com enxerto de \\
fíbula
\end{tabular} & Sangue \\
\hline $\begin{array}{c}\text { Mannarino et } \\
\text { al. (2014) } \\
\text { Brasil }\end{array}$ & Série de casos & $(2 \overbrace{}^{2} \mathrm{e} 0$ o & $15-20$ & $\begin{array}{l}\text { Cisto ósseo } \\
\text { simples }\end{array}$ & 1 & 0 & $\begin{array}{c}\text { Mandíbula } \\
\text { (RP): } 2 \\
\text { (RA): } 0\end{array}$ & 0 & 2 & 0 & 1 & 1 & $\begin{array}{l}\text { Exploração } \\
\text { cirúrgica e } \\
\text { curetagem }\end{array}$ & $\begin{array}{c}\text { Cavidade vazia } \\
\text { e sangue }\end{array}$ \\
\hline $\begin{array}{c}\text { Ribeiro, Bisol, } \\
\text { Iwaki, Silva, } \\
\text { \& Tolentino } \\
(2014) \\
\text { Brasil } \\
\end{array}$ & Série de casos & $\left(20^{3} \mathrm{e} 1\right.$ 1 $)$ & $16-22$ & $\begin{array}{l}\text { Cisto ósseo } \\
\text { simples }\end{array}$ & 0 & 0 & $\begin{array}{c}\text { Mandíbula } \\
\text { (RP): } 2 \\
\text { (RA):1 }\end{array}$ & 0 & 3 & 0 & 3 & 0 & $\begin{array}{l}\text { Exploração } \\
\text { cirúrgica }\end{array}$ & Sangue \\
\hline $\begin{array}{c}\text { Iwaki et al. } \\
\text { (2016) } \\
\text { Brasil }\end{array}$ & $\begin{array}{c}\text { Coorte } \\
\text { retrospectivo }\end{array}$ & $\begin{array}{c}12 \\
\left(4{ }^{3} \mathrm{e} 8 \text { 年) }\right.\end{array}$ & $13-40$ & $\begin{array}{l}\text { Cisto ósseo } \\
\text { solitário }\end{array}$ & $*$ & 0 & $\begin{array}{l}\text { Mandíbula } \\
\text { (RP): } 8 \\
\text { Mandíbula } \\
\text { (RA): } 4\end{array}$ & 1 & 12 & 0 & 9 & 3 & $\begin{array}{l}\text { Exploração } \\
\text { cirúrgica e } \\
\text { curetagem }\end{array}$ & * \\
\hline $\begin{array}{c}\text { You, Kim, \& } \\
\text { Ahn (2017) } \\
\text { Coreia do Sul }\end{array}$ & $\begin{array}{c}\text { Coorte } \\
\text { retrospectivo }\end{array}$ & 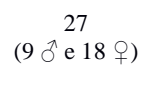 & $10-63$ & $\begin{array}{c}\text { Cavidade óssea } \\
\text { idiopática }\end{array}$ & 2 & 0 & $\begin{array}{c}\text { Mandíbula } \\
\text { (RP): } 21 \\
\text { (RA): } 5\end{array}$ & $*$ & 25 & 2 & $*$ & $*$ & $\begin{array}{l}\text { Exploração } \\
\text { cirúrgica e } \\
\text { curetagem }\end{array}$ & $*$ \\
\hline
\end{tabular}


Research, Society and Development, v. 9, n. 10, e109107656, 2020

(CC BY 4.0) | ISSN 2525-3409 | DOI: http://dx.doi.org/10.33448/rsd-v9i10.7656

\begin{tabular}{|c|c|c|c|c|c|c|c|c|c|c|c|c|c|c|}
\hline & & & & & & & Maxila:1 & & & & & & & \\
\hline $\begin{array}{c}\text { Battisti et al. } \\
(2018) \\
\text { Brasil } \\
\end{array}$ & $\begin{array}{c}\text { Coorte } \\
\text { prospectivo }\end{array}$ & $\left(\begin{array}{c}21 \\
\left(8 \mathrm{o}^{\lambda} \mathrm{e} 13 \text { ? }\right.\end{array}\right.$ & $11-30$ & $\begin{array}{c}\text { Cavidade óssea } \\
\text { idiopática }\end{array}$ & * & 1 & $\begin{array}{c}\text { Mandíbula } \\
\text { (RP): } 9 \\
\text { (RA): } 12\end{array}$ & 0 & 19 & 2 & * & * & Proservação & * \\
\hline $\begin{array}{l}\text { Razmara, } \\
\text { Ghoncheh, \& } \\
\text { Shabankare } \\
\text { (2019) } \\
\text { Iran }\end{array}$ & Série de casos & $\left(0 \stackrel{3}{3} \mathrm{e}^{3}\right.$ \& $)$ & $13-23$ & $\begin{array}{l}\text { Cisto ósseo } \\
\text { traumático }\end{array}$ & 1 & 0 & $\begin{array}{l}\text { Mandíbula } \\
\text { (RP): } 2 \\
\text { (RA): } 1\end{array}$ & 1 & 3 & 0 & 3 & 0 & $\begin{array}{l}\text { Exploração } \\
\text { cirúrgica }\end{array}$ & $\begin{array}{c}\text { Cavidade vazia } \\
\text { e sangue }\end{array}$ \\
\hline
\end{tabular}

*Dados não verificados no estudo ou não mencionados de forma clara pelos autores.

Fonte: Tabela confeccionada pelos autores. 
De acordo com a Tabela 2 é possível observar, de maneira quantitativa, os tipos de estudo, amostra, idade, tipo de lesão, histórico de trauma orofacial, sintomatologia, localização das lesões, presença de aumento volumétrico em ossos faciais acometidos, características radiográficas (unilateral, bilateral, unilocular, multilocular), achados intraoperatórios, terapêutica instituída e presença ou ausência recidiva das lesões.

Dentre os 21 estudos inclusos, a maioria foi de coorte retrospectivo, com amostra total de 401 pacientes (193 do sexo masculino e 208 do sexo feminino) e idade mínima de 7 anos e máxima de 75 anos. O diagnóstico das lesões intraósseas segue a seguinte distribuição de acordo com o quantitativo de pacientes: cisto ósseo traumático (210), cisto ósseo simples (100), cavidade óssea idiopática (57), cisto ósseo de extravasamento (15) cisto ósseo solitário (12) e cisto ósseo aneurismático (7). Com relação a amostra total, o histórico de trauma orofacial anterior ao diagnóstico das lesões esteve presente em 83 pacientes, enquanto a sintomatologia álgica ocorreu em 43.

A localização das lesões ocorreu de acordo com o seguinte quantitativo de pacientes: região posterior de mandíbula (268), região anterior de mandíbula (104) e maxila (30). Em 40 pacientes houve expansão óssea. Com relação às características radiográficas, 369 pacientes apresentaram lesões com distribuição unilateral e 29 com localização bilateral, enquanto o padrão unilocular esteve presente em 97 e o padrão multilocular em 23 pacientes. A maioria das lesões foi abordada por meio de exploração cirúrgica e curetagem, com o achado transoperatório mais frequente consistindo em cavidade vazia ou com ar, seguido da presença de sangue e líquido seroso. Embora mencionada de maneira não muito clara, a recidiva das lesões ocorreu de forma não significativa.

\section{Discussão}

Inicialmente descrito como cisto ósseo traumático (Lucas, 1929), a cavidade óssea idiopática é uma lesão rara com patogênese incerta que afeta os ossos maxilares. Sua etiologia pode estar vinculada a várias hipóteses: trauma mecânico, anormalidades vasculares, degeneração cística de tumores ósseos, necrose isquêmica da medula óssea, alterações no metabolismo do cálcio, a, alterações nas funções dos osteoclastos e na remodelação óssea (Blum,1955; Whinery, 1955).

De acordo com a teoria traumático-hemorrágica, a falta de resolução ou lise do coágulo sanguíneo intramedular após o trauma pode estimular sua formação (Howe, 1965). O trauma facial apresenta maior incidência em jovens do sexo masculino (Montovani et al., 2006), 
embora os resultados do nosso estudo mostrem que dos 210 pacientes diagnosticados com cisto ósseo traumático, apenas 61 apresentaram histórico de trauma e a presença de sangue no interior da cavidade óssea foi infrequente, fortalecendo a incerteza quanto a fisiopatologia dessa lesão (Bhoosreddy, Gadgil, Bhoosreddy, \& Valenkiwar, 2010; Harnet et al., 2008; Homem de Carvalho, Martins, Carrad, \& Rados, 2010; Teoh, Watts, Chee, Reid, \& Porter, 2010). O seu interior pode apresentar-se vazio, conter sangue ou líquido serossanguíneo. À medida que a lesão envelhece, o conteúdo de fluidos tende a diminuir, a cavidade fica vazia, o que permite a cura espontânea da lesão. No entanto, não há uma explicação clara para a falha precoce na organização do hematoma (Mathew, Gianoli, Omami, \& Lurie 2012).

Pode ocorrer em qualquer idade, embora seja mais frequente durante as primeiras duas décadas de vida (Forssell et al., 1988). MacDonald-Jankowaki (1995), sugere que a ocorrência reduzida em pacientes mais velhos esteja relacionado a natureza autorreparadora da COI. Assim como já descrito na literatura, os resultados da presente revisão também não mostram diferença significativa na ocorrência de COI entre pacientes do sexo masculino e feminino. Ocorre de forma quase exclusiva em regiões posteriores da mandíbula, com menor frequência na sínfise e raramente na maxila (Tong et al., 2003). Essa distribuição pode ter explicação pela teoria trumático-hemorrágica, pois por tratar-se de um osso cortical, pode haver menor capacidade de reparação na mandíbula quando comparado a maxila (Martinsfilho et al., 2012).

Geralmente assintomática e raramente com expansão de cortical óssea, a COI é frequentemente diagnosticada em radiografias panorâmicas de rotina (Kumar, Sherubin, Raman, \& Shettar, 2011), concordando com os resultados do presente estudo, pois em um universo de 401 pacientes houve sintomatologia em apenas 43. A escassez de relatos de expansão cortical pode ser um indício de desenvolvimento e propagação desta lesão por espaços medulares (Martins-Filho et al., 2012). Entretanto, diante episódios de infecção secundária pode haver dor, edema e/ou perda da função (Strabbing, Gortzak, Vinke, Saridin, \& Van Merkesteyn, 2011).

Radiograficamente, a COI caracteriza-se como uma lesão radiolúcida unilocular com margens festonadas envolvendo raízes dentárias em região posterior da mandíbula, apesar de também haver lesões com aspecto radiográfico multilocular (Perdigão, Silva, Sakurai, Soares de Araújo, \& Gomes, 2003). Dessa forma, a COI deve ser incluída no diagnóstico diferencial de lesões como cisto dentígero, tumor odontogênico queratocístico, mixoma odontogênico, ameloblastoma, cisto radicular e lesão central de células gigantes, cisto ósseo aneurismático, defeito focal da medula óssea e malformação vascular (Cowan, 1980; Shigematsu et al., 
19942. Devido a escassez de material para análise histopatológica, o diagnóstico definitivo da COI geralmente é realizado no momento da exploração cirúrgica. Durante o transoperatório, a lesão pode abrigar uma cavidade vazia com ar, sangue e/ou líquido seroso (Hansen, Sapone, \& Sproat, 1974; Discacciati et al., 2012).

Dentre as terapêuticas cirúrgicas disponíveis para a COI, a curetagem das paredes ósseas é o método mais utilizado (Suei, Taguchi, \& Tanimoto, 2007). Os resultados do presente estudo mostraram que este foi o tratamento de escolha para a maioria dos pacientes, sendo efetivo e com recidiva ocorrendo apenas alguns pacientes, principalmente em casos de lesões multiloculares e/ou bilaterais (Forssell et al., 1988; Matsummura et al., 1998; You, Kim, \& Ahn, 2017).

\section{Conclusão}

Os resultados do presente estudo indicam que a cavidade óssea idiopática encontra-se de forma predominante durante as primeiras duas décadas de vida de pacientes de ambos os sexos, tem maior incidência em região posterior de mandíbula, é geralmente assintomática, radiograficamente apresentando-se de forma incisiva como uma lesão radiolúcida unilocular com ar, sangue ou líquido seroso no seu interior, tendo a exploração cirúrgica e curetagem como tratamento de escolha e baixo índice de recidiva.

Sugere-se que mais estudos sejam realizados com o intuito de estabelecer parâmetros clínicos de maior segurança, desde o diagnóstico ao manejo terapêutico da cavidade óssea idiopática em ossos gnáticos. Além disso, é importante estabelecer o tempo de acompanhamento pós-operatório considerado ideal no qual não ocorra recidiva e possa determinar cura do paciente.

\section{Referências}

An, S. Y., Lee J. S., Benavides, E., Aminlari A., McDonald, N. J., Edwards, P. S., Heo, M. S., Shin, H. I., Park, J. W., Jung, J. K., Choi, K. S., \& An, C. H. (2014). Multiple simple bone cysts of the jaws: review of the literature and report of three cases. Oral Surgery, Oral Medicine, Oral Pathology and Oral Radiology, 117( 6), 458-469. 
Battisti, M. P. L, Soares, M. Q. S., Rubira, C. M. F., Bullen, I. R. F. R., Lauris, J. R. P., \& Damante, J. H. (2018). Assessment of spontaneus resolution of idiopathic bone cavity. Journal of Applied Oral Science, 26, 1-5.

Beasley, J. D. (1976). Traumatic cyst of the jaws: report of 30 cases. Journal of The American Dental Association, 92(1), 145-152.

Blum, T. (1955). Symposium: an additional report on traumatic bone cysts. Oral Surgery, Oral Medicine, and Oral Pathology, 8(9), 917-939.

Bhoosreddy, A. R., Gadgil, R. M., Bhoosreddy, S. A., \& Valenkiwar, G. N. (2010). Solitary bone cyst: a case report and review of literature. Journal of Indian Academy of Oral Medicine and Radiology, 22(1), 27-29.

Cortell-Ballester, I., Figueiredo R., Beirini-Aytés, L., \& Gay-Escoda, C. (2009). Traumatic bone cyst: a retrospective study of 21 cases. Medicina oral, patologia oral y cirurgia bucal, 14(5), 239-243.

Cowan, C. G. (1980). Traumatic bone cysts of the jaws and their presentation. International Journal of Oral Surgery, 9(4), 287-291.

Davis Junior, W. M., Buchs, A. U., \& Davis, W. M. (1979). Extravasation cyst diagnostic curettement: A treatment. Oral Surgery, Oral Medicine, and Oral Pathology, 47(1), 2-7.

Discacciati, E. D., Faria, V. M. C., Garcia, N. G., Sakai, V. T., Pereira, A. A. C., \& Hanemann, J., A. C. (2012). Idiopathic bone cavity: case series involving children and adolescents. Journal of Investigative and Clinical Dentistry, 3(2), 103-108.

Forssell, K., Forssell, H., Happonen, R. P., \& Neva, M. (1988). Simple bone cyst. Review of the literature and analysis of 23 cases. International Journal of Oral and Maxillofacial Surgery, 17(1), 21-24.

Hansen, L. S., Sapone, J., \& Sproat, R. C. (1974). Traumatic bone cysts of jaws: Report of sixty-six cases. Oral Surgery, Oral Medicine, Oral Pathology, 37(6), 899-910. 
Harnet, J. C., Lombardi, T., Klewansky, P., Rieger, J., Tempe, M. H., \& Clavert, J. M. (2008). Solitary bone cyst of the jaws:a review of the etiopathogenic hypotheses. Journal of Oral and Maxillofacial Surgery, 66(11), 2345-2348.

Homem de Carvalho, A. L., Martins, M. D., Carrad, V. C., \& Rados, P. V. (2010). Simple bone cyst: report of cases and proposal for a minimal surgical intervention. International Journal of Pediatric Otorhinolaryngology, 74(12), 1449-1451.

Howe, G. L. (1965). "Haemorrhagic cysts" of the mandible. I. The British Journal of Oral Surgery, 3(1), 55-76.

Iwaki, L. C. V, Iwaki-Filho, L., Takeshita, W. M., Chicarelli, M., Preis, L. A. M., \& Bragatto, F. P. (2016). Solitary bone cyst: an analysis of 12 cases. Acta Scientiarum, 38(1), 103-109.

Kaugars, G. E. \& Cale, A. E. (1987). Traumatic bone cyst. Oral Surgery, Oral Medicine, and Oral Pathology, 63(3), 318-324.

Kumar, N. D., Sherubin, J. E., Raman, U., \& Shettar, S. (2011). Solitary bone cyst. Indian Journal of Dental Research, 22(1), 172-174.

Lindsay, J. S., Martin, W. R., \& Green, H. G. (1966). Traumatic bone cyst treated with homogenous bone graft. Report of a case. Oral Surgery, Oral Medicine, and Oral Pathology, 21(4), 536-542.

Lucas, C.D. (1929). Do all cysts of the jaws originate from the dental system? The Journal of the American Dental Association, 16(4), 647-661.

Macdonald-Janckowski, D. S. (1995). Traumatic bone cysts in the jaws of a Hong Kong Chinese Population. Clinical Radiology, 50, 787-791.

Mannarino, F. S., Gorla, L. F. O., Gabrilli, M. F. R., Vieira, E. H., Gabrielli, M. A. C., \& Pereira-Filho, V. A. (2014). Cisto ósseo simples-relatos de casos. Revista de Cirurgia e Traumatologia Buco-maxilo-facial, 14(3), 15-20. 
Mathew, R., Gianoli, D., Omami, G., \& Lurie, A. (2012). Unusual cone-beam computerized tomography presentation of traumatic (simple) bone cyst: case report and radiographic analysis. Oral Surgery, Oral Medicine, Oral Pathology, Oral Radiology and Endodontology, 113(3), 410-413.

Martins-Filho, P. R. S., Santos, T. S., Araújo, V. L. C., Santos, J. S., Andrade, E. S. S., \& Silva, L. C. F. (2012). Traumatic bone cyst of the mandible: a review of 26 cases. Brazilian Journal of Otorhinolaryngology, 78(2), 16-21.

Matsumura, S., Murakami, S., Kakimoto, N., Kishino, M., Ishida, T., \& Fuchihata, H. (1998). Histopathologic and radiographic findings of the simple bone cyst. Oral Surgery, Oral Medicine, Oral Pathology, Oral Radiology and Endodontics, 85(5), 619-625.

Montovani, J. C., Campos, L. M. P., Gomes, M. A., Moraes, V. R. S., Ferreira, F. D., \& Nogueira, E. A. (2006). Etiologia e incidência das fraturas faciais em adultos e crianças: experiência em 513 casos. Revista Brasileira de Otorrinolaringologia, 72(2), 235-241.

Paes, B. L. L., Santos, K. C. P., Marucci, M., Costa, C., \& Oliveira, J. X. (2010). Cisto ósseo simples: avaliação radiográfica, anatomopatológica e clínica de seis casos, Journal of the Health Sciences Institute, 28(1), 71-76.

Peñarrocha-Diago, M., Sanchis-Bielsa, J. M., Bonet-Marco, J., \& Minguez-Sanz, J. M. (2001). Surgical tretment and follow-up of solitary bone cyst of the mandible: a report of seven cases, The British of Oral and Maxillofacial Surgery, 39(3), 221-223.

Perdigão, P. F., Silva, E. C., Sakurai, E., Soares de Araújo, N., \& Gomes, R. S. (2003). Idiopathic boné cavity: a clinical, radiographic, and histological study. British Journal of Oral and Maxillofacial Surgery, 41, 407-409.

Razmara, F., Ghoncheh, Z., \& Shabankare, G. (2019). Traumatic bone cyst of mandible: a case series. Journal of Medical Case Reports, 13(1), 1-8. 
Ribeiro, E. B., Bisol, F. C. T., Iwaki, L. C. V., Silva, M. C., \& Tolentino, E. S. (2014). Cisto ósseo simples: relato de casos clínicos. Revista da Faculdade de Odontologia Universidade de Passo Fundo, 19(3), 359-363.

Rushton, M. A. (1946). Solitary bone cysts in the mandible. British Dental Journal, 81(2), 37 49.

Sabino-Bezerra, J. R., Santos-Silva, A. R., Jorge, J. Gouvêa, A. F., \& Lopes, M. A. (2013). Atypical presentations of simple bone cysts of the mandible: a case series and review of literature, Journal of Cranio-maxillo-facial Surgery, 41(5), 391-396.

Shigematsu, H., Fujita, K., \& Watanabe, K. (1994). Atypical simple bone cyst of the mandible. International Journal of Oral and Maxillofacial Surgery, 23(5), 298-299.

Shimoyama, T., Horie, N., Nasu, D., Kaneko, T., Kato, T., Tojo, T., Suzuki T., \& Ide, F. (1999). So-called simple bone cyst of the jaw:a family of pseudocysts of diverse nature and etiology. Journal of Oral Science. 41(2), 93-98.

Strabbing, E. M., Gortzak, R. A., Vinke, J. G., Saridin, C. P., \& Van Merkesteyn, J. P. (2011). An atypical presentation of a solitary bone cyst of the mandibular ramus: a case report. Journal of Craniomaxillofacial Surgery, 39(2), 45-47.

Suei, Y., Taguchi, A., \& Tanimoto, K. (2007). Simple bone cyst of the jaws:evaluation of treatment outcome by review of 132 cases. Journal of Oral and Maxillofacial Surgery, 65(5), 918-923.

Teoh, K. H., Watts, A. C., Chee, Y. H., Reid, R., \& Porter, D. E. (2010) . Predictive factors for recurrence of simple bone cyst of the proximal humerus. Journal of Orthopaedic Surgery, 18(2), 215-219.

Tong, A. C., Ng, I. O., \& Yan, B. S. (2003). Variations in clinical presentations of the simple bone cyst: report of cases. Journal of Oral and Maxillofacial Surgery, 61(12), 1487-1491. 
Tsukamoto, G., Sasaki, A., Mese, H., \& Matsumara, T. (2003). Atypical Simple Bone Cyst of the Jaws - Multiple Cysts and Repeated Recurrence. Asian Journal of Oral Maxillofacial Surgery, 15(2), 138-141.

Urs, A. B., Augustine, J., \& Chawla H. (2014). Aneurysmal Bone Cyst os the Jaws: Clinicopathological Study. Journal of Maxillofacial and Oral Surgery, 13(14), 458-463.

Wakasa, T., Kawai, N., Aiga, H., \& Kishi, K. (2002). Management of florid cementoosseous dysplasia of the mandible producing solitary bone cyst:report of a case. Journal of Oral and Maxillofacial Surgery, 60(7), 832-835.

Whinery, J. G. (1955) Symposium: solitary bone cysts of the mandible. Oral Surgery, Oral Medicine, and Oral Pathology, 8(9), 903-916.

You, M. S., Kim, D. Y., \& Ahn, K. M. (2017). Surgical management of idiopathic bone cavity: case series of consecutive 27 patients. Journal of the Korean Association of Oral and Maxillofacial Surgeons, 43(2), 94-99.

\title{
Porcentagem de contribuição de cada autor no manuscrito
}

\author{
Vinícius Lima de Almeida - 40\% \\ Cristóvão Marcondes de Castro Rodrigues - 15\% \\ Marco Túllio Brazão-Silva - 10\% \\ Flávio de Freitas Bueno - 10\% \\ Maiolino Thomaz Fonseca Oliveira $-25 \%$
}

